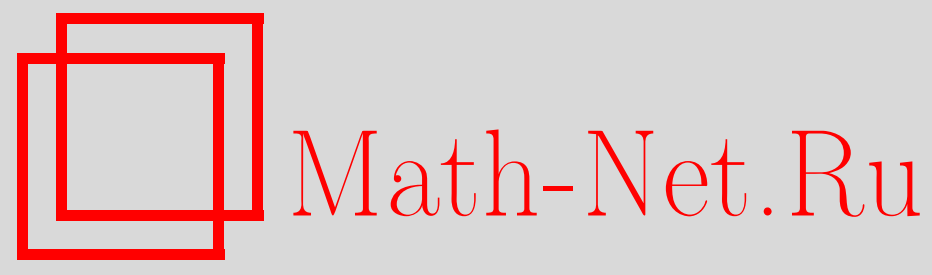

В. И. Хомич, K вопросу об отделимости нормализуемых суперинтуиционистских пропозициональных логик, Матем. заметки, 1998, том 64, выпуск 4, 606-615

DOI: https://doi.org/10.4213/mzm1436

Использование Общероссийского математического портала Math-Net.Ru подразумевает, что вы прочитали и согласны с пользовательским соглашением http://www.mathnet.ru/rus/agreement

Параметры загрузки:

IP : 52.205 .19 .152

26 апреля 2023 г., 14:10:53 


\section{К ВОПРОСУ ОБ ОТДЕЛИМОСТИ \\ НОРМАЛИЗУЕМЫХ СУПЕРИНТУИЦИОНИСТСКИХ ПРОПОЗИЦИОНАЛЬНЫХ ЛОГИК}

\section{В.И. Хомич}

В работе изучается проблема отделимости суперинтуиционистских пропозициональных логик - расширений интуиционистской пропозициональной логики. Получен критерий отделимости нормальных суперинтуиционистских пропозициональных исчислений, а также результаты, касающиеся полноты фрагментов таких исчислений. Данный критерий позволяет устанавливать отделимость нормализуемых суперинтуиционистских пропозициональных логик. С помощью этих результатов исправлены обнаруженные автором ошибки в доказательствах некоторых утверждений Мак-Кея и Хосои.

Библиографоия: 26 названий.

Настоящая работа посвящена изучению проблемы отделимости суперинтуиционистских пропозициональньх логик - расширений интуиционистской пропозициональной логики (в зарубежной литературе суперинтуиционистские пропозициональные логики назьвают промежуточными пропозициональными логиками - intermediate propositional logics). Мы строим для заданной логики равнообъемное с ней исчисление гильбертова типа, удовлетворяющее следующим условиям:

1) каждая его аксиома содержит не более двух логических знаков, причем если их два, то один из них - импликация;

2) для любой выводимой в этом исчислении формулы существует ее вывод в нем, формулы которого не содержат логических знаков, отличньх от импликации и знаков рассматриваемой формулы.

Свойство отделимости исчислений, определяемое условиями 1) и 2), введено в статье [1], там же доказана отделимость интуиционистской пропозициональной логики. Однако, предложенное доказательство содержит ошибку [2, с. 399], что стало предметом обсуждения в работах [3]-[5]. Впервые правильное доказательство отделимости этой логики дано в [6] (см. также [7, с. 406]). В [7] доказана отделимость и классической пропозициональной логики. В [8] отделимость интуиционистской пропозициональной логики получена алгебраическим методом. Далее, в работах [9]-[12] доказана отделимость табличных логик, порожденных конечными и счетной линейно упорядоченньми псевдобулевьми алгебрами.

Работа выполнена при финансовой поддержке Российского фонда фундаментальных исследований, грант № 94-01-00944. 
Впервые пример неотделимой логики был построен в статье [13]. Неотделимость той логики состоит в невозможности задать ее исчислением, удовлетворяющим условию 1). Чтобы констатировать наличие или отсутствие этого факта, там же были введены понятия нормального исчисления и нормализуемой логики. Исчисление называется нормальным, если оно удовлетворяет условию 1), а логика - нормализуемой, если ее можно задать нормальньм исчислением. Ненормализуемые логически и, следовательно, неотделимые были обнаружены среди табличных логик [14], [15].

В работах [15]-[19] автором рассматривалась проблема отделимости суперинтуиционистских пропозициональных логик, порожденных формулами, не содержащими дизъюнкции или допускающими ее элиминирование. Такие логики можно задать исчислениями, дополнительные аксиомы которых не содержат логических знаков, отличных от импликации и отрицания. Для них условно решена проблема отделимости [18], что позволило доказать отделимость логик из достаточно широкого их класса, содержащего логики, порожденные импликативными формулами [18], [19]. Для четырех нетривиальных классов исчислений построены алгоритмы, распознающие отделимые среди исчислений этих классов [17], [19].

Вместе с тем в работах [13], [20], [21] исследовалась проблема отделимости логик, допускающих задание в виде нормальных исчислений, дополнительные аксиомы которых содержат знак дизъюнкции. Отметим, что для этой цели не применимы методы, разработанные автором в [15]-[19].

В настоящей работе изучается проблема отделимости нормализуемых суперинтуиционистских пропозициональных логик. Получен критерий (теорема 1) отделимости нормальных суперинтуиционистских пропозициональных исчис лений, позволяющий устанавливать отделимость и нормализуемых суперинтуиционистских пропозициональных логик. С помощью теоремы 1 исправлена обнаруженная автором ошибка (см. [17]) в доказательстве теоремы 3 из [13] об отделимости нормализуемых табличных логик и, тем самым, устранены пробелы в доказательствах следствий 2.6, 3.7 и 4.13 из [20]. Кроме того, получены результаты (теорема 2, следствие 1), касающиеся полноты фрагментов исчислений, дополнительные аксиомы которых не содержат логических знаков, отличных от импликации и отрицания; изложены также результаты статьи [21] (найдена ошибка в доказательстве леммы 5.2, эта лемма легко следует из теоремы 16 работы [18], и опровергнута лемма 5.4, исправлением которой является теорема 1).

1. Введем необходимые понятия и обозначения. Интуииионистское пропозициональное исчисление [7], [8], заданное десятью аксиомами и двумя правилами вывода (подстановка и модус поненс), будем обозначать через $\boldsymbol{H}$, а суперинтуиционистское пропозичиональное исчисление, получающееся из $\boldsymbol{H}$ путем добавления в список аксиом $\boldsymbol{H}$ конечного множества пропозициональных формул $\mathfrak{X}$, назьваемых его дополнительными аксиомами, - через $\boldsymbol{H}+\mathfrak{X}$. Исчисления $\boldsymbol{H}+\mathfrak{X}$ и $\boldsymbol{H}+\mathfrak{H}$ назьваются равнообъемными, если множество формул, выводимых в $\boldsymbol{H}+\mathfrak{X}$, совпадает с множеством формул, выводимых в $\boldsymbol{H}+\mathfrak{H}$.

Пусть $\mu$ - какой-нибудь набор логических пропозициональных знаков. Пропозициональную формулу назовем $\mu$-фор мулой, если она не содержит логических знаков, отличных от знаков набора $\mu$; $\mu$-формула назьвается $\mu$-выводимой в $\boldsymbol{H}+\mathfrak{X}$, если в $\boldsymbol{H}+\mathfrak{X}$ существует ее вывод, каждая формула которого является $\mu$-формулой. Исчисление $\boldsymbol{H}+\mathfrak{X}$ назьвается нормальным [13], если каждая его аксиома является $\supset \neg$-формулой, или

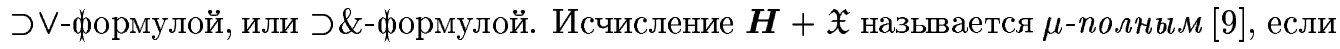


всякая $\mu$-формула, выводимая в $\boldsymbol{H}+\mathfrak{X}$, является $\mu \cup\{\supset\}$-выводимой в нем. Исчисление $\boldsymbol{H}+\mathfrak{X}$ называется отделимым [1], [8], [7], [22], [9], если $\boldsymbol{H}+\mathfrak{X}$ является нормальным и $\mu$-полньм при любом $\mu$.

Согласно работе [8] аксиомы интуиционистского пропозиционального исчисления можно выбрать так, что оно будет отделимым. Поэтому в дальнейшем мы будем считать, что $\boldsymbol{H}$ является отделимым исчислением.

Суперинтуиционистской пропозициональной логикой называется множество пропозициональных формул, содержащее все аксиомы $\boldsymbol{H}$ и замкнутое относительно правила подстановки и правила модус поненс. Суперинтуиционистское пропозициональное исчисление назьвается аксиоматизацией суперинтуиционистской пропозициональной логики, если в нем вьводимы все ее формулы и только они. Суперинтуиционистская пропозициональная логика называется аксиоматизируемой (нормализуемой, отделимой [13]), если существует ее аксиоматизация (нормальная аксиоматизация, отделимая аксиоматизация соответственно).

Пусть $\nu$ - набор логических пропозициональных знаков, содержащий импликацию. Понятие $\nu$-алгебры, введенное в работе [8] для изучения фрагментов исчисления $\boldsymbol{H}$, стало обобщением таких уже известных под другими названиями понятий, как импликативная полуструктура, импликативная структура и псевдобулева алгебра [23], [24]. По сути дела, $\nu$-алгебра - это множество, на котором заданы операции псевдобулевой алгебры, определяемые набором $\nu$ и включающие операцию относительного псевдодополнения. Точнее говоря, $\nu$-алгебра - это множество $\Xi$, в котором указан выделенньй элемент, обозначаемый через $\mathbf{1}$, и заданы соответствуюшие логическим знакам набора $\nu$ операции, удовлетворяющие следующим условиям:

1) если для любого $\xi$ из $\Xi$ верно $\mathbf{1} \supset \xi=\mathbf{1}$, то имеет место $\xi=\mathbf{1}$;

2) если для любых $\xi$ и $\eta$ из $\Xi$ верны $\xi \supset \eta=\mathbf{1}$ и $\eta \supset \xi=\mathbf{1}$, то имеет место $\xi=\eta$;

3 ) для каждой $\nu$-аксиомы $Q$ (т.е. аксиомы, являющейся $\nu$-формулой) исчисления $\boldsymbol{H}$ значение любого выражения, получающегося из $Q$ путем замены переменных $\nu$-формулы $Q$ элементами $\Xi$, а логических знаков соответствующими им операциями $\Xi$, равно выделенному элементу.

Операции $\nu$-алгебр обозначаем так же, как и соответствующие им (интерпретируемые ими) логические пропозициональные связки.

Определение $\nu$-алгебры можно найти также в работе [19]. При $\nu$, содержащем все четыре логических пропозициональных знака, $\nu$-алгебра будет псевдобулевой алгеброй.

Множество пропозициональных формул, верных (общезначимых) на какой-либо конечной псевдобулевой алгебре, является суперинтуиционистской пропозициональной логикой, которую назьвают табличной логикой.

В работах [25], [13] понятия суперинтуиционистской пропозициональной логики и табличной логики обобщены следующим образом. Суперинтуиционистской $\nu$-логикой назьвается множество $\nu$-формул, содержащее все $\nu$-аксиомы $\boldsymbol{H}$ и замкнутоеотносительно правила модус поненс и правила подстановки $\nu$-формул. Множество $\nu$-формул, верных на какой-нибудь конечной $\nu$-алгебре, является суперинтуиционистской $\nu$-логикой, которую называют табличной $\nu$-логикой. Для любой суперинтуиционистской пропозициональной логики (табличной логики) $\mathfrak{Q}$ подмножество $\mathfrak{Q} \nu$ всех $\nu$-формул $\mathfrak{Q}$ является суперинтуиционистской $\nu$-логикой (табличной $\nu$-логикой соответственно). Естественньм образом понятия аксиоматизируемости и нормализуемости суперинтуиционистских пропозициональных логик распространяются [25], [13] и на $\nu$-логики. 
Пусть $\mathfrak{X}$ - конечное непустое множество пропозициональных формул, $Y, Z, X_{1}, \ldots, X_{n}$ - какие-нибудь пропозициональные формулы, а $q_{1}, \ldots, q_{n}$ - различные пропозициональные переменные. Выражение $Y \vdash Z(Y \models Z)$ означает, что формула $Z$ вьводима в $\boldsymbol{H}$ из формулы $Y$ с помошью обоих правил (с помошью правила модус поненс и вьводимых в $\boldsymbol{H}$ формул соответственно); выражение $\vdash Z$ означает, что формула $Z$ вьводима в $\boldsymbol{H}$, выражение $\vdash^{\nu} Z$ означает, что $\nu$-формула $Z \nu$-выводима в $\boldsymbol{H}$, а выражение $\boldsymbol{F}\left(q_{1}, \ldots, q_{n}\left|X_{1}, \ldots, X_{n}\right| Y\right)$ - результат подстановки в формулу $Y$ вместо переменных $q_{1}, \ldots, q_{n}$ формул $X_{1}, \ldots, X_{n}$ соответственно.

Конъюнкцию всех элементов множества $\mathfrak{X}$ обозначим через $\overline{\mathfrak{X}}$, а формулу $X_{n} \supset(\cdots \supset$ $\left.\left(X_{1} \supset Z\right) \cdots\right)$ - через $\left\{X_{1}, \ldots, X_{n}\right\} \supset Z$.

2. Докажем критерий отделимости нормальных суперинтуиционистских пропозициональных исчислений.

ТеОрема 1. Пусть $\mathfrak{X}$ - конечное множество формул, каждая из которых яв-

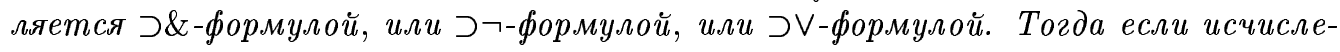

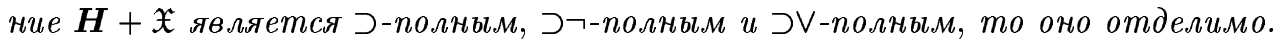

ДокАЗАТЕЛЬСТво. Пусть задано конечное множество $\mathfrak{X}$ формул, каждая из которых является $\supset \&$-формулой, или $\supset$-формулой, или $\supset \vee$-формулой. Если $\mathfrak{X}=\varnothing$, то теорема верна.

Пусть $\mathfrak{X} \neq \varnothing$ и исчисление $\boldsymbol{H}+\mathfrak{X}$ является つ-полным, つ ᄀ-полным и $\supset$-полным. Согласно определению отделимости исчислений нам достаточно доказать $\mu$-полноту исчисления $\boldsymbol{H}+\mathfrak{X}$ при $\mu$, содержащем $\supset$ и не содержащем все четыре логических пропозициональных знака. Пусть $\nu$ - один из следуюших наборов логических знаков: $\supset$, или $\supset \neg$, или $\supset \vee$. Тогда с помощью результатов работы [13] нетрудно показать, что исчисление $\boldsymbol{H}+\mathfrak{X}$ будет $\nu \&$-полным. Тем не менее, докажем этот факт.

Пусть $\nu \&$-формула $Q$ выводима в исчислении $\boldsymbol{H}+\mathfrak{X}$. Тогда с помощью следующих выводимых в $\boldsymbol{H}$ эквивалентностей:

$$
\begin{gathered}
((X \& Y) \supset Z) \equiv(X \supset(Y \supset Z)), \quad(X \supset(Y \& Z)) \equiv((X \supset Y) \&(X \supset Z)), \\
((X \& Y) \vee Z) \equiv((X \vee Z) \&(Y \vee Z)), \quad(X \vee(Y \& Z)) \equiv((X \vee Y) \&(X \vee Z)), \\
\neg(X \& Y) \equiv(X \supset \neg Y),
\end{gathered}
$$

где $X, Y$ и $Z$ - произвольные формулы, для $Q$ построим непустое конечное множество $\nu$-формул $\mathfrak{H}$ такое, что $\vdash Q \equiv \overline{\mathfrak{H}}$. Поэтому имеем $\vdash \overline{\mathfrak{H}} \supset Q$, значит верна вьводимость $\vdash \mathfrak{H} \supset Q$. Так как формула $\mathfrak{H} \supset Q$ является $\nu \&$-формулой, в силу отделимости $\boldsymbol{H}$ имеем $\vdash^{\nu \&} \mathfrak{H} \supset Q$. Но формула $\overline{\mathfrak{H}}$ выводима в $\boldsymbol{H}+\mathfrak{X}$. Тогда и все формулы множества $\mathfrak{H}$ выводимы в $\boldsymbol{H}+\mathfrak{X}$. Так как каждая из них является $\nu$-формулой и исчисление $\boldsymbol{H}+\mathfrak{X}$ $\nu$-полное, все они $\nu$-вьводимы в $\boldsymbol{H}+\mathfrak{X}$. Поэтому $\nu \&$-формула $Q \nu \&$-выводима в $\boldsymbol{H}+\mathfrak{X}$. Следовательно, исчисление $\boldsymbol{H}+\mathfrak{X}$ является $\nu \&$-полным.

Покажем, что исчисление $\boldsymbol{H}+\mathfrak{X}$ будет $\mu$-полньм при $\mu$ равном $\supset \vee \neg$. Пусть $\mu$-формула $S$ выводима в $\boldsymbol{H}+\mathfrak{X}$. Если $\vdash S$, то, используя непустоту $\mathfrak{X}$, получаем, что $\vdash \boldsymbol{F}(q|q| P) \supset S$, где $p \in \mathfrak{X}$, а $q$-пропозициональная переменная. Если $S$ невьводима в $\boldsymbol{H}$, то в силу непустоты $\mathfrak{X}$ каждый вывод формулы $S$ в $\boldsymbol{H}+\mathfrak{X}$ содержит аксиомы из $\mathfrak{X}$. Тогда верна выводимость $\vdash \&_{i=1}^{n} \boldsymbol{F}\left(q_{1}^{i}, \ldots, q_{m_{i}}^{i}\left|Q_{1}^{i}, \ldots, Q_{m_{i}}^{i}\right| P_{i}\right) \supset S$, где $P_{i} \in \mathfrak{X}(1 \leqslant i \leqslant n), q_{1}^{i}, \ldots, q_{m_{i}}^{i}-$ 
различные пропозициональные переменные, а $Q_{1}^{i}, \ldots, Q_{m_{i}}^{i}$ - формулы. Так же, как и выше, для каждой формулы $Q_{j}^{i}\left(1 \leqslant i \leqslant n, 1 \leqslant j \leqslant m_{i}\right)$ построим непустое конечное множество $\mu$-формул $\mathfrak{X}_{j}^{i}$ такое, что $\vdash Q_{j}^{i} \equiv \overline{\mathfrak{X}}_{j}^{i}$. Тогда имеем $\vdash \&_{i=1}^{n} \boldsymbol{F}\left(q_{1}^{i}, \ldots, q_{m_{i}}^{i} \mid \overline{\mathfrak{X}}_{1}^{i}, \ldots\right.$, $\left.\overline{\mathfrak{X}}_{m_{i}}^{i} \mid P_{i}\right) \supset S$.

Пусть $\mathfrak{Z}_{1}^{1}, \ldots, \mathfrak{Z}_{m_{1}}^{1}, \ldots, \mathfrak{Z}_{1}^{n}, \ldots, \mathfrak{Z}_{m_{n}}^{n}$ - множества пропозициональных переменных такие, что все их переменные отличны друг от друга, число переменных множества $\mathfrak{Z}_{j}^{i}$ $\left(1 \leqslant i \leqslant n, 1 \leqslant j \leqslant m_{i}\right.$ ) равно числу формул множества $\mathfrak{X}_{j}^{i}$ (т.е. $\left.\left|\mathfrak{Z}_{j}^{i}\right|=\left|\mathfrak{X}_{j}^{i}\right|\right)$, и все переменные из множеств $\mathfrak{Z}_{1}^{i}, \ldots, \mathfrak{Z}_{m_{i}}^{i}(1 \leqslant i \leqslant n)$ не входят в формулу $P_{i}$. Так как $P_{i}-\nu$-формула, то $\boldsymbol{F}\left(q_{1}^{i}, \ldots, q_{m_{i}}^{i}\left|\overline{\mathfrak{Z}}_{1}^{i}, \ldots, \overline{\mathfrak{Z}}_{m_{i}}^{i}\right| P_{i}\right)$ является $\nu \&$-формулой. Для каждой такой $\nu \&$-формулы построим непустое конечное множество $\nu$-формул $\mathfrak{U}_{i}$ такое, что $\vdash \boldsymbol{F}\left(q_{1}^{i}, \ldots, q_{m_{i}}^{i}\left|\overline{\mathfrak{Z}}_{1}^{i}, \ldots, \overline{\mathfrak{Z}}_{m_{i}}^{i}\right| P_{i}\right) \equiv \overline{\mathfrak{U}}_{i}$. Поэтому

$$
\begin{aligned}
& \vdash \boldsymbol{F}\left(\mathfrak{Z}_{1}^{i}, \ldots, \mathfrak{Z}_{m_{i}}^{i}\left|\mathfrak{X}_{1}^{i}, \ldots, \mathfrak{X}_{m_{i}}^{i}\right| \boldsymbol{F}\left(q_{1}^{i}, \ldots, q_{m_{i}}^{i}\left|\overline{\mathfrak{Z}}_{1}^{i}, \ldots, \overline{\mathfrak{Z}}_{m_{i}}^{i}\right| P_{i}\right)\right) \\
& \equiv \overline{\left\{\boldsymbol{F}\left(\mathfrak{Z}_{1}^{i}, \ldots, \mathfrak{Z}_{m_{i}}^{i}\left|\mathfrak{X}_{1}^{i}, \ldots, \mathfrak{X}_{m_{i}}^{i}\right| Y\right): Y \in \mathfrak{U}_{i}\right\}} \text {. }
\end{aligned}
$$

Так как все переменные из множеств $\mathfrak{Z}_{1}^{i}, \ldots, \mathfrak{Z}_{m_{i}}^{i}$ отличны друг от друга и не входят в формулу $P_{i}$, то

$$
\begin{aligned}
& \boldsymbol{F}\left(\mathfrak{Z}_{1}^{i}, \ldots, \mathfrak{Z}_{m_{i}}^{i}\left|\mathfrak{X}_{1}^{i}, \ldots, \mathfrak{X}_{m_{i}}^{i}\right| \boldsymbol{F}\left(q_{1}^{i}, \ldots, q_{m_{i}}^{i}\left|\overline{\mathfrak{Z}}_{1}^{i}, \ldots, \overline{\mathfrak{Z}}_{m_{i}}^{i}\right| P_{i}\right)\right) \\
& \quad=\boldsymbol{F}\left(q_{1}^{i}, \ldots, q_{m_{i}}^{i}\left|\overline{\mathfrak{X}}_{1}^{i}, \ldots, \overline{\mathfrak{X}}_{m_{i}}^{i}\right| P_{i}\right) .
\end{aligned}
$$

Следовательно, $\vdash\left\{\boldsymbol{F}\left(\mathfrak{Z}_{1}^{i}, \ldots, \mathfrak{Z}_{m_{i}}^{i}\left|\mathfrak{X}_{1}^{i}, \ldots, \mathfrak{X}_{m_{i}}^{i}\right| Y\right): Y \in \mathfrak{U}_{i}, 1 \leqslant i \leqslant n\right\} \supset S$. Поскольку эта формула является $\mu$-формулой, в силу отделимости $\boldsymbol{H}$ имеем $\vdash^{\mu}\left\{\boldsymbol{F}\left(\mathfrak{Z}_{1}^{i}, \ldots, \mathfrak{Z}_{m_{i}}^{i} \mid\right.\right.$ $\left.\left.\mathfrak{X}_{1}^{i}, \ldots, \mathfrak{X}_{m_{i}}^{i} \mid Y\right) \mid Y \in \mathfrak{U}_{i}, 1 \leqslant i \leqslant n\right\} \supset S$.

Легко видеть, что формула $\boldsymbol{F}\left(q_{1}^{i}, \ldots, q_{m_{i}}^{i}\left|\overline{\mathfrak{Z}}_{1}^{i}, \ldots, \overline{\mathfrak{Z}}_{m_{i}}^{i}\right| P_{i}\right)$ вьводима в $\boldsymbol{H}+\mathfrak{X}$. Тогда и формула $\overline{\mathfrak{U}}_{i}$ выводима в $\boldsymbol{H}+\mathfrak{X}$. Поэтому все формулы множества $\mathfrak{U}_{i}$ выводимы в $\boldsymbol{H}+\mathfrak{X}$. Так как $\nu$ есть $\supset$, или $\supset$, или $\supset \vee, \mathfrak{U}_{i}-$ множество $\nu$-формул, а исчисление $\boldsymbol{H}+\mathfrak{X} \nu$-полное, то все $\nu$-формулы множества $\mathfrak{U}_{i} \nu$-выводимы в $\boldsymbol{H}+\mathfrak{X}$. Но $\nu \subseteq \mu$ и для любого $j\left(1 \leqslant j \leqslant m_{i}\right) \mathfrak{X}_{j}^{i}$ является множеством $\mu$-формул. Тогда все формулы множества $\left\{\boldsymbol{F}\left(\mathfrak{Z}_{1}^{i}, \ldots, \mathfrak{Z}_{m_{i}}^{i}\left|\mathfrak{X}_{1}^{i}, \ldots, \mathfrak{X}_{m_{i}}^{i}\right| Y\right): Y \in \mathfrak{U}_{i}, 1 \leqslant i \leqslant n\right\}$ будут $\mu$-формулами, $\mu$-вьводимьми в $\boldsymbol{H}+\mathfrak{X}$. Поэтому $\mu$-формула $S \mu$-выводима в $\boldsymbol{H}+\mathfrak{X}$. Следовательно, исчисление $\boldsymbol{H}+\mathfrak{X}$ является $\mu$-полным. Теорема 1 доказана.

Из теоремы 1 следует, что любая суперинтуиционистская пропозициональная логика, имеющая нормальную, つ-полную, つᄀ-полную и $\supset$-полную аксиоматизацию, отде-

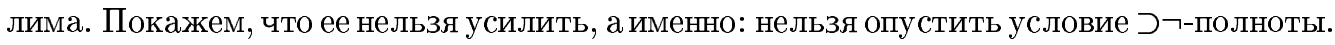
Для этого достаточно построить нормальное исчисление, являющееся つ-полнњм и $\supset \vee$-полным и не являющееся つᄀ-полным.

Пусть $q, t$ и $w$-пропозициональные переменные. Формулу $(\neg \neg q \supset t) \supset(((\neg \neg q \supset q) \supset$ $t) \supset t$ ) обозначим через $P$, а исчисление $\boldsymbol{H}+\{P\}$ - через $\mathfrak{T}$. Очевидно, что исчисление $\mathfrak{T}$ является нормальным. Так как имеет место вьводимость $\neg q \vee \neg \neg q \models \neg \neg q \vee(\neg \neg q \supset q)$, в силу результатов работы [25] или [26] множество つ \&\&-формул, выводимых в $\boldsymbol{H}+$ $\{\neg \neg q \vee(\neg \neg q \supset q)\}$, совпадает с множеством $\supset \vee \&$-формул, вьводимых в $\boldsymbol{H}$. Очевидно, верны выводимости $\neg \neg q \vee(\neg \neg q \supset q) \vDash P$ и $\boldsymbol{F}(t|\neg \neg q \vee(\neg \neg q \supset q)| P) \mid \models \neg \neg q \vee(\neg \neg q \supset q)$. 
Тогда исчисления $\mathfrak{T}$ и $\boldsymbol{H}+\{\neg \neg q \vee(\neg \neg q \supset q)\}$ равнообъемны. Поэтому множество $\supset \vee \&$-формул, выводимых в $\mathfrak{T}$, совпадает с множеством $\supset \vee \&$-формул, выводимых в $\boldsymbol{H}$. Поскольку исчисление $\boldsymbol{H}$ отделимо, исчисление т будет つ-полным и $\supset \vee$-полным.

Докажем, что исчисление $\mathfrak{T}$ не является つᄀ-полным (факт анонсирован в работе [15]). Для этой цели достаточно показать, что $\supset$-формула

$$
T=(\neg \neg w \supset t) \supset(((\neg \neg w \supset(\neg \neg q \supset q)) \supset t) \supset t)
$$

выводима в $\boldsymbol{H}+\{\neg \neg q \vee(\neg \neg q \supset q)\}$, но не $\supset \neg$-выводима в $\mathfrak{T}$, т.е. не существует ее вывода в $\mathfrak{T}$, состоящего только из $\supset$-формул.

Легко видеть, что $\neg \neg q \vee(\neg \neg q \supset q) \vdash \neg \neg(w \& q) \vee(\neg \neg(w \& q) \supset(w \& q))$. Кроме того, в $\boldsymbol{H}$ выводима эквивалентность

$$
\begin{aligned}
& (\neg \neg(w \& q) \vee(\neg \neg(w \& q) \supset(w \& q))) \\
& \quad \equiv(((\neg \neg w \& \neg \neg q) \vee((\neg \neg w \& \neg \neg q) \supset w)) \&((\neg \neg w \& \neg \neg q) \vee((\neg \neg w \& \neg \neg q) \supset q))) .
\end{aligned}
$$

Тогда верна выводимость $\neg \neg q \vee(\neg \neg q \supset q) \vdash(\neg \neg w \& \neg \neg q) \vee((\neg \neg w \& \neg \neg q) \supset q)$. Нетрудно проверить, что $(\neg \neg w \& \neg \neg q) \vee((\neg \neg w \& \neg \neg q) \supset q) \vDash T$. Поэтому $\neg \neg q \vee(\neg \neg q \supset q) \vdash T$. Следовательно, $T$ выводима в $\boldsymbol{H}+\{\neg \neg q \vee(\neg \neg q \supset q)\}$.

Покажем, что $\supset \neg$-формула $T$ не будет $\supset$-вьводимой в $\mathfrak{T}$. Достаточно построить конечную $\supset$-алгебру, на которой $\supset$-формула $P$ верна, а $\supset$-формула $T$ опровержима. Рассмотрим псевдобулеву алгебру $\Omega$ с диаграммой

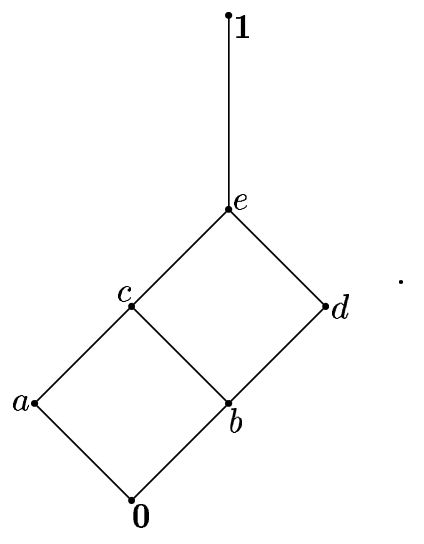

Легко видеть, что множество $\Phi=\{\mathbf{0}, a, b, c, d, e, \mathbf{1}\}$ элементов $\Omega$ замкнуто в $\Omega$ относительно операций $\supset$ и $\neg$, а следовательно, является $\supset$-алгеброй.

Покажем, что $\supset$-формула $P$ верна на $\Phi$. Пусть задана оценка $f$ переменных $\supset$-формулы $P$ на $\Phi$. Рассмотрим случай $f(q) \in\{\mathbf{0}, a, d\}$. Тогда $\neg \neg f(q)=f(q)$, откуда

$$
\begin{aligned}
f(P) & =(\neg \neg f(q) \supset f(t)) \supset(((\neg \neg f(q) \supset f(q)) \supset f(t)) \supset f(t)) \\
& =(f(q) \supset f(t)) \supset(((f(q) \supset f(q)) \supset f(t)) \supset f(t)) \\
& =(f(q) \supset f(t)) \supset((\mathbf{1} \supset f(t)) \supset f(t))=(f(q) \supset f(t)) \supset(f(t) \supset f(t)) \\
& =(f(q) \supset f(t)) \supset \mathbf{1}=\mathbf{1} .
\end{aligned}
$$


Рассмотрим случай $f(q) \in\{c, e, \mathbf{1}\}$. Тогда $\neg \neg f(q)=\mathbf{1}$, поэтому

$$
\begin{aligned}
f(P) & =(\neg \neg f(q) \supset f(t)) \supset(((\neg \neg f(q) \supset f(q)) \supset f(t)) \supset f(t)) \\
& =(\mathbf{1} \supset f(t)) \supset(((\mathbf{1} \supset f(q)) \supset f(t)) \supset f(t))=f(t) \supset((f(q) \supset f(t)) \supset f(t))=\mathbf{1} .
\end{aligned}
$$

Покажем, что $\supset$-формула $T$ опровержима на $\Phi$. Построим оценку $g$ переменных $\supset$ ᄀ-формулы $T$ на $\Phi$, полагая $g(q)=c, g(t)=e$ и $g(w)=d$. Тогда

$$
\begin{aligned}
g(T) & =(\neg \neg d \supset e) \supset(((\neg \neg d \supset(\neg \neg c \supset c)) \supset e) \supset e) \\
& =(d \supset e) \supset(((d \supset(\mathbf{1} \supset c)) \supset e) \supset e)=\mathbf{1} \supset(((d \supset c) \supset e) \supset e) \\
& =((d \supset c) \supset e) \supset e=(c \supset e) \supset e=\mathbf{1} \supset e=e .
\end{aligned}
$$

Таким образом, исчисление $\mathfrak{T}$ является нормальньм, つ-полным и $\supset$ V-полным и не является $\supset$-полным. Значит, в теореме 1 нельзя опустить условие $\supset \neg$-полноты. Заметим, что нельзя опустить хотя бы одно из условий つ-полноты, или $\supset$-полноты, или $\supset \vee$-полноты и, тем самым, нельзя усилить теорему 1.

В работе [17] в доказательстве теоремы 3 из [13] обнаружен пробел. С помощью теоремы 1 установим справедливость этой теоремы.

ТЕОремА 2. Любая нормализуемая табличная логика отделима.

ДокАЗАТЕЛЬСТво. Пусть задана табличная нормализуемая логика $\mathfrak{Q}$. Тогда существует конечное множество формул $\mathfrak{X}$ такое, что исчисление $\boldsymbol{H}+\mathfrak{X}$ будет аксиоматизацией логики $\mathfrak{Q}$ и каждая формула $\mathfrak{X}$ является $\supset$-формулой, или $\supset$-формулой, или $\supset$-формулой. Пусть $\nu$ - один из следующих наборов логических знаков: $\supset$, или $\supset \vee$, или $\supset$ ᄀ. Легко видеть, что множество $\mathfrak{Q}_{\nu}$ всех $\nu$-формул табличной логики $\mathfrak{Q}$ будет табличной $\nu$-логикой. Так же, как в теореме 3 из [13], с помощью результатов работы [25] построим аксиоматизацию табличной $\nu$-логики $\mathfrak{Q}_{\nu}$. Согласно построению этой аксиоматизации множество всех ее аксиом $\mathfrak{X}^{\nu}$ конечно и содержит только $\nu$-формулы. Положим $\mathfrak{H}=\mathfrak{X} \cup \mathfrak{X} \supset \cup \mathfrak{X} \supset \vee \cup \mathfrak{X} \supset$. Нетрудно проверить, что исчисление $\boldsymbol{H}+\mathfrak{H}$ является нормальной аксиоматизацией логики $\mathfrak{Q}$. В силу построения исчисления $\boldsymbol{H}+\mathfrak{H}$

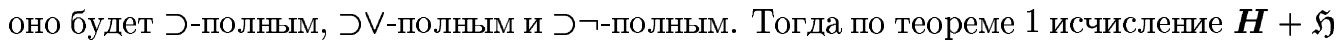
отделимо. Следовательно, логика $\mathfrak{Q}$ отделима. Теорема 2 доказана.

Таким образом, теорема 3 из [13] оказьвается верной и, значит, справедливы следствия 2.6, 3.7 и 4.13 из [20], полученные с помощью этой теоремы.

3. Используя идею доказательства теоремы 1 , докажем следующее утверждение.

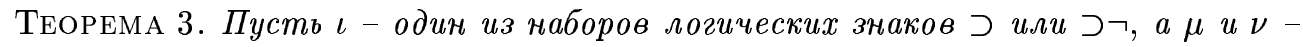

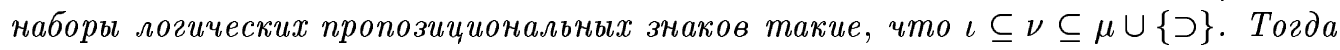
если для любых конечных мнохсеств ь-формул $\mathfrak{X}$ и $\mathfrak{H}$ исчисления $\boldsymbol{H}+\mathfrak{X} u \boldsymbol{H}+\mathfrak{H}$ являются $\nu$-полными, то исчисление $\boldsymbol{H}+(\mathfrak{X} \cup \mathfrak{H})$ будет $\mu$-полным.

ДокАЗАТЕЛЬСТво. Пусть заданы наборы логических знаков $\iota, \mu$ и $\nu$, удовлетворяющие условию теоремы. Возьмем конечные множества $\iota$-формул $\mathfrak{X}$ и $\mathfrak{H}$ такие, что исчисления $\boldsymbol{H}+\mathfrak{X}$ и $\boldsymbol{H}+\mathfrak{H}$ являются $\nu$-полньпи. Если $\mathfrak{X} \cup \mathfrak{H}=\varnothing$, то согласно отделимости $\boldsymbol{H}$ исчисление $\boldsymbol{H}+(\mathfrak{X} \cup \mathfrak{H})$ будет $\mu$-полньм.

Пусть $\mathfrak{X} \cup \mathfrak{H} \neq \varnothing$. Если \& $\in \mu$, то в силу [17, следствие 1 и лемма 1] исчисление $\boldsymbol{H}+(\mathfrak{X} \cup \mathfrak{H})$ будет $\mu$-полным. Пусть $\& \notin \mu$. Докажем, что и в этом случае исчисление 
$\boldsymbol{H}+(\mathfrak{X} \cup \mathfrak{H})$ будет $\mu$-полньм. Для простоты и удобства изложения набор логических знаков $\mu \cup\{\supset\}$ обозначим через $\tau$. Пусть $\mu$-формула $Q$ выводима в $\boldsymbol{H}+(\mathfrak{X} \cup \mathfrak{H})$. Так как $\mu \subseteq \tau$, в силу [17, следствие 1 и лемма 1$] \mu$-формула $Q \tau \&$-выводима в $\boldsymbol{H}+(\mathfrak{X} \cup \mathfrak{H})$. Используя непустоту $\mathfrak{X} \cup \mathfrak{H}$, так же, как и в теореме 1 получаем, что $\vdash \&_{i=1}^{n} \boldsymbol{F}\left(q_{1}^{i}, \ldots, q_{m_{i}}^{i} \mid\right.$ $\left.S_{1}^{i}, \ldots, S_{m_{i}}^{i} \mid P_{i}\right) \supset Q$, где $P_{i} \in \mathfrak{X} \cup \mathfrak{H}(1 \leqslant i \leqslant n), q_{1}^{i}, \ldots, q_{m_{i}}^{i}$ - различные пропозициональные переменные, а $S_{1}^{i}, \ldots, S_{m_{i}}^{i}-\tau \&$-формулы. Для каждой $\tau \&$-формулы $S_{j}^{i}$ $\left(1 \leqslant i \leqslant n, 1 \leqslant j \leqslant m_{i}\right)$ построим непустое конечное множество $\tau$-формул $\mathfrak{X}_{j}^{i}$ такое, что $\vdash S_{j}^{i} \equiv \overline{\mathfrak{X}}_{j}^{i}$. Тогда $\vdash \&_{i=1}^{n} \boldsymbol{F}\left(q_{1}^{i}, \ldots, q_{m_{i}}^{i}\left|\overline{\mathfrak{X}}_{1}^{i}, \ldots, \overline{\mathfrak{X}}_{m_{i}}^{i}\right| P_{i}\right) \supset Q$.

Пусть $\mathfrak{Z}_{1}^{1}, \ldots, \mathfrak{Z}_{m_{1}}^{1}, \ldots, \mathfrak{Z}_{1}^{n}, \ldots, \mathfrak{Z}_{m_{n}}^{n}$ - множества пропозициональных переменных такие, что все их переменные отличны друг от друга, число переменных множества $\mathfrak{Z}_{j}^{i}$ $\left(1 \leqslant i \leqslant n, 1 \leqslant j \leqslant m_{i}\right.$ ) равно числу формул множества $\mathfrak{X}_{j}^{i}$ (т.е. $\left|\mathfrak{Z}_{j}^{i}\right|=\left|\mathfrak{X}_{j}^{i}\right|$ ), и все переменные из множеств $\mathfrak{Z}_{1}^{i}, \ldots, \mathfrak{Z}_{m_{i}}^{i}(1 \leqslant i \leqslant n)$ не входят в формулу $P_{i}$. Так как $P_{i}$ - $\iota$-формула, то $\boldsymbol{F}\left(q_{1}^{i}, \ldots, q_{m_{i}}^{i}\left|\overline{\mathfrak{Z}}_{1}^{i}, \ldots, \overline{\mathfrak{Z}}_{m_{i}}^{i}\right| P_{i}\right)$ является $\iota \&$-формулой. Для каждой такой $\iota \&$-формулы построим непустое конечное множество $\iota$-формул $\mathfrak{U}_{i}$ такое, что $\vdash \boldsymbol{F}\left(q_{1}^{i}, \ldots, q_{m_{i}}^{i}\left|\overline{\mathfrak{Z}}_{1}^{i}, \ldots, \overline{\mathfrak{Z}}_{m_{i}}^{i}\right| P_{i}\right) \equiv \overline{\mathfrak{U}}_{i}$. Тогда

$$
\begin{aligned}
& \vdash \boldsymbol{F}\left(\mathfrak{Z}_{1}^{i}, \ldots, \mathfrak{Z}_{m_{i}}^{i}\left|\mathfrak{X}_{1}^{i}, \ldots, \mathfrak{X}_{m_{i}}^{i}\right| \boldsymbol{F}\left(q_{1}^{i}, \ldots, q_{m_{i}}^{i}\left|\overline{\mathfrak{Z}}_{1}^{i}, \ldots, \overline{\mathfrak{Z}}_{m_{i}}^{i}\right| P_{i}\right)\right) \\
& \quad \equiv \frac{\left.\boldsymbol{F}\left(\mathfrak{Z}_{1}^{i}, \ldots, \mathfrak{Z}_{m_{i}}^{i}\left|\mathfrak{X}_{1}^{i}, \ldots, \mathfrak{X}_{m_{i}}^{i}\right| Y\right): Y \in \mathfrak{U}_{i}\right\}}{} .
\end{aligned}
$$

Поскольку все переменные из множеств $\mathfrak{Z}_{1}^{i}, \ldots, \mathfrak{Z}_{m_{i}}^{i}$ отличны друг от друга и не входят в формулу $P_{i}$, то

$$
\begin{aligned}
& \boldsymbol{F}\left(\mathfrak{Z}_{1}^{i}, \ldots, \mathfrak{Z}_{m_{i}}^{i}\left|\mathfrak{X}_{1}^{i}, \ldots, \mathfrak{X}_{m_{i}}^{i}\right| \boldsymbol{F}\left(q_{1}^{i}, \ldots, q_{m_{i}}^{i}\left|\overline{\mathfrak{Z}}_{1}^{i}, \ldots, \overline{\mathfrak{Z}}_{m_{i}}^{i}\right| P_{i}\right)\right) \\
& \quad=\boldsymbol{F}\left(q_{1}^{i}, \ldots, q_{m_{i}}^{i}\left|\overline{\mathfrak{X}}_{1}^{i}, \ldots, \overline{\mathfrak{X}}_{m_{i}}^{i}\right| P_{i}\right) .
\end{aligned}
$$

Следовательно, $\vdash\left\{\boldsymbol{F}\left(\mathfrak{Z}_{1}^{i}, \ldots, \mathfrak{Z}_{m_{i}}^{i}\left|\mathfrak{X}_{1}^{i}, \ldots, \mathfrak{X}_{m_{i}}^{i}\right| Y\right): Y \in \mathfrak{U}_{i}, 1 \leqslant i \leqslant n\right\} \supset Q$. Так как эта формула является $\tau$-формулой, в силу отделимости $\boldsymbol{H}$ имеем $\vdash^{\tau}\left\{\boldsymbol{F}\left(\mathfrak{Z}_{1}^{i}, \ldots, \mathfrak{Z}_{m_{i}}^{i} \mid\right.\right.$ $\left.\left.\mathfrak{X}_{1}^{i}, \ldots, \mathfrak{X}_{m_{i}}^{i} \mid Y\right): Y \in \mathfrak{U}_{i}, 1 \leqslant i \leqslant n\right\} \supset Q$.

Легко видеть, что формула $\boldsymbol{F}\left(q_{1}^{i}, \ldots, q_{m_{i}}^{i}\left|\overline{\mathfrak{Z}}_{1}^{i}, \ldots, \overline{\mathfrak{Z}}_{m_{i}}^{i}\right| P_{i}\right)$ вьводима в $\boldsymbol{H}+\mathfrak{X}$ или $\boldsymbol{H}+\mathfrak{H}$. Тогда и формула $\overline{\mathfrak{U}}_{i}$ выводима в $\boldsymbol{H}+\mathfrak{X}$ или $\boldsymbol{H}+\mathfrak{H}$. Поэтому все формулы множества $\mathfrak{U}_{i}$ выводимы в $\boldsymbol{H}+\mathfrak{X}$ или все формулы множества $\mathfrak{U}_{i}$ вьводимы в $\boldsymbol{H}+\mathfrak{H}$. Так как $\iota \subseteq \nu, \mathfrak{U}_{i}-$ множество $\iota$-формул, а исчисления $\boldsymbol{H}+\mathfrak{X}$ и $\boldsymbol{H}+\mathfrak{H} \nu$-полные, то все $\nu$-формулы множества $\mathfrak{U}_{i} \nu$-выводимы в $\boldsymbol{H}+\mathfrak{X}$ или все $\nu$-формулы множества $\mathfrak{U}_{i}$ $\nu$-вьводимы в $\boldsymbol{H}+\mathfrak{H}$. Но $\nu \subseteq \tau$ и для любого $j\left(1 \leqslant j \leqslant m_{i}\right) \mathfrak{X}_{j}^{i}$ является множеством $\tau$-формул. Тогда все формулы множества $\left\{\boldsymbol{F}\left(\mathfrak{Z}_{1}^{i}, \ldots, \mathfrak{Z}_{m_{i}}^{i}\left|\mathfrak{X}_{1}^{i}, \ldots, \mathfrak{X}_{m_{i}}^{i}\right| Y\right): Y \in \mathfrak{U}_{i}\right.$, $1 \leqslant i \leqslant n\}$ будут $\tau$-формулами, $\tau$-выводимыми в $\boldsymbol{H}+(\mathfrak{X} \cup \mathfrak{H})$. Поэтому $\mu$-формула $Q$ $\tau$-выводима в $\boldsymbol{H}+(\mathfrak{X} \cup \mathfrak{H})$. Следовательно, исчисление $\boldsymbol{H}+(\mathfrak{X} \cup \mathfrak{H})$ является $\mu$-полным. Теорема 3 доказана.

Теорема 3 показьвает, что $\mu$-полнота рассматриваемых исчислений сохраняется с расширением $\mu$. Кроме того, из нее вытекает

СлЕДСТВИЕ 1. Если для любых конечных множсеств つ-формул $\mathfrak{X}$ и $\mathfrak{H}$ исчисления $\boldsymbol{H}+\mathfrak{X} u \boldsymbol{H}+\mathfrak{H}$ являются つ-полными, то исчисление $\boldsymbol{H}+(\mathfrak{X} \cup \mathfrak{H})$ отделимо.

Если исчисления $\boldsymbol{H}+\{P\}$ и $\boldsymbol{H}+\{Q\}$, где $P$ и $Q-\supset$-формулы, отделимы, то согласно следствию 1 исчисление $\boldsymbol{H}+\{P, Q\}$ также отделимо. Таким методом можно доказать отделимость многих исчис лений. 
4. В работе [21] рассматривался вопрос об отделимости суперинтуиционистских пропозициональных логик, множество $\supset \vee \&$-формул которых совпадает с множеством

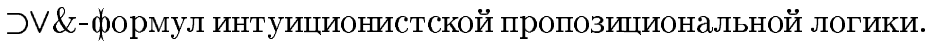

Лемма [21, лемма 5.2]. Для кажсдой такой логики $\mathfrak{G}$ следующие условия әквивалентны:

1) логика $\mathfrak{G}$ обладает свойством отделимости;

2) логика $\mathfrak{G}$ мохет быть аксиоматизирована путем добавления некоторых $\supset \neg$-аксиом к интуииионистской системе;

3) логика $\mathfrak{G}$ может быть аксиоматизирована посредством つ-аксиом, つ\&-ак-

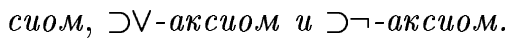

Несколько расплывчатую формулировку этой леммы уточняет ее доказательство, основную часть которого составляет проверка истинности импликации 2) $\Longrightarrow 1$ ), где по существу доказьвается отделимость любого исчисления $\boldsymbol{H}+\mathfrak{X}(\mathfrak{X}-$ произвольное

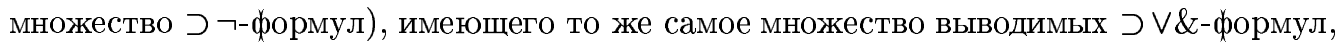
что и $\boldsymbol{H}$. Опровергнем это утверждение.

Рассмотрим логику $\mathfrak{L}(\neg \neg q \vee(\neg \neg q \supset q))$, т.е. множество формул, выводимых в исчислении $\boldsymbol{H}+\{\neg \neg q \vee(\neg \neg q \supset q)\}$. Так как имеет место выводимость $\neg q \vee \neg \neg q \models \neg \neg q \vee$

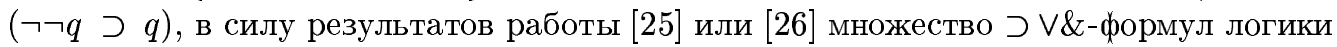

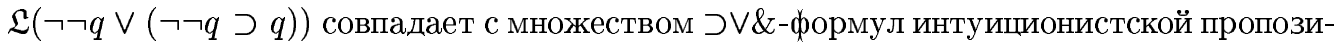
циональной логики. Поэтому логика $\mathfrak{L}(\neg \neg q \vee(\neg \neg q \supset q))$ попадает в область действия леммы 5.2 из [21]. Легко видеть, что исчисление $\mathfrak{T}$ является аксиоматизацией логики $\mathfrak{L}(\neg \neg q \vee(\neg \neg q \supset q))$, удовлетворяющей условию 2). Согласно доказанному вьше исчисление $\mathfrak{T}$ не является つᄀ-полньм и, следовательно, не будет отделимым.

Покажем, что из теоремы 16 работы [18] легко следует лемма 5.2 из [21]. С этой целью напомним определение введенного в [17] свойства $\mathfrak{P}_{\nu}$ для суперинтуиционистских пропозициональных исчислений, где $\nu$ - набор логических пропозициональных знаков, не содержащий ᄀ. Будем говорить, что исчисление $\boldsymbol{H}=\mathfrak{X}$ обладает свойством $\mathfrak{P}_{\nu}$, если каждая вьводимая в $\boldsymbol{H}+\mathfrak{X} \boldsymbol{\nu}$-формула вьводима и в исчислении $\boldsymbol{H}+\mathfrak{X} \supset \vee \&$.

Нетрудно установить верность импликаций 1) $\Longrightarrow 3$ ) и 3$) \Longrightarrow 2$ ). Докажем импликацию 2) $\Longrightarrow$ 1). Пусть исчисление $\boldsymbol{H}+\mathfrak{Z}$, где $\mathfrak{Z}$ - конечное множество $\supset$-формул, является аксиоматизацией логики $\mathfrak{G}$. Легко видеть, что оно обладает свойством $\mathfrak{P} \supset \vee \cdot$ Тогда по теореме 16 из [18] для $\mathfrak{Z}$ существует конечное множество $\supset \neg$-формул $\mathfrak{B}$ такое, что исчисление $\boldsymbol{H}+\mathfrak{B}$ отделимо и равнообъемно исчислению $\boldsymbol{H}+\mathfrak{Z}$.

В лемме 5.4 из [21] утверждается, что для отделимости произвольной суперинтуиционистской пропозициональной логики достаточно существования еенормальной, つ-полной и つV-полной аксиоматизации. По существу в ней доказьвается отделимость любого нормального, つ-полного и $\supset \bigvee$-полного суперинтуиционистского пропозиционального исчисления. Так как исчисление $\mathfrak{T}$ является нормальным, つ-полным и $\supset$-полным и не является つᄀ-полным, то лемма 5.4 из [21] неверна. Ееисправлением является теорема 1.

\section{СПИСОК ЦИТИРОВАННОЙ ЛИТЕРАТУРЫ}

[1] Wajsberg M. Untersuchungen über den Aussagenkalkül von A. Heyting // Wiadom. Mat. 1938. V. 46. P. 45-101.

[2] Черч А. Введение в математическую логику. Т. 1. М.: ИЛ, 1960. 
[3] Kabziǹski J.K. The Wajsberg's results connected with separability of the intuitionistic propositional logic // Bull. Sect. Logic. Univ. Łódź. 1973. V. 2. № 2. P. 131-133.

[4] Kabziǹski J. K., Porebska M. Proof of the separability of the intuitionistic propositional logic by the Wajsberg method // Rep. Math. Logic. 1975. V. 4. P. 31-38.

[5] Циткин А. И. К вопросу об ошшбке в известной работе М. Вайсберга // Исследования по неклассическим логикам и теории множеств. М.: Наука, 1979. С. 240-256.

[6] Curry H. A note on the reduction of Gentzen's calculus LJ // Bull. Amer. Math. Soc. 1939. V. 45. № 4. P. 288-293.

[7] Клини С. К. Введение в метаматематику. М.: ИЛ, 1957.

[8] Horn A. The separation theorem of intuitionist propositional calculus // J. Symbolic Logic. 1962. V. 27. № 4. P. 391-399.

[9] Hosoi T. On the separation theorem of intermediate propositional calculi // Proc. Japan Acad. Ser. A. Math. Sci. 1966. V. 42. №6. P. 535-538.

[10] Hosoi T. Algebraic proof of the separation theorem on Dummett's LC // Proc. Japan Acad. Ser. A. Math. Sci. 1966. V. 42. № 7. P. 693-695.

[11] Hosoi T. The separable axiomatization of the intermediate propositional systems $S_{n}$ of Gödel // Proc. Japan Acad. Ser. A. Math. Sci. 1966. V. 42. №9. P. 1001-1006.

[12] Hosoi T. A criterion for the separable axiomatization of Gödel's $S_{n}$ // Proc. Japan Acad. Ser. A. Math. Sci. 1967. V. 43. № 5. P. 365-368.

[13] McKay C. G. The non-separability of a certain finite extension of Heyting's propositional logic // Proc. Konink. Nederl. Akad. Wetensch. 1968. V. 71. № 3. P. 312-315.

[14] Hosoi T. Non-separable intermediate propositional logics // J. Tsuda College. 1976. V. 8. P. 13-18.

[15] Хомич В.И. Отделимость суперинтуиционистских пропозициональных логик // Исследования по теории алгорифмов и математической логике. М.: Наука, 1979. С. 98-115.

[16] Хомич В. И. Теорема отделимости для суперинтуиционистских исчислений высказываний // Докл. АН СССР. 1976. Т. 229. №6. С. 1327-1329.

[17] Хомич В.И. О проблеме отделимости для суперинтуиционистских пропозициональных логик // Докл. АН СССР. 1980. Т. 254. № 4. С. 820-823.

[18] Хомич В.И. Об отделимых суперинтуиционистских пропозициональных исчислениях и о конъюнктивно неразложимых элементах в импликативных полуструктурах // Z. Math. Logik Grundlag. Math. 1986. V. 32. № 2. P. 149-180.

[19] Хомич В.И. О свойствах суперинтуиционистских пропозициональных исчислений // Сиб. матем. ж. 1990. Т. 31. №6. С. 158-175.

[20] Hosoi T., Ono H. Axiomatization of models for intermediate logics constructed with Boolean models by piling up // Publ. Res. Inst. Math. Sci. 1972. V. 8. № 1. P. 1-11.

[21] Hosoi T. On intermediate logics. III // J. Tsuda College. 1974. V. 6. P. 23-38.

[22] Hosoi T. The separation theorem on the classical system // J. Fac. Sci. Univ. Tokyo. Sect. IA. Math. 1966. V. 12. №2. P. 223-230.

[23] Карри Х. Б. Основания математической логики. М.: Мир, 1969.

[24] Расева Е., Сикорский Р. Математика метаматематики. М.: Наука, 1972.

[25] McKay C. G. The decidability of certain intermediate propositional logics // J. Symbolic Logic. 1968. V. 33. № 2. P. 258-264.

[26] Янков В. А. Об исчислении слабого закона исключенного третьего // Изв. АН СССР. Сер. матем. 1968. Т. 32. № 5. С. 1044-1051. 